\title{
VISIÓN DE LA UNMSM AL AÑO 2021
}

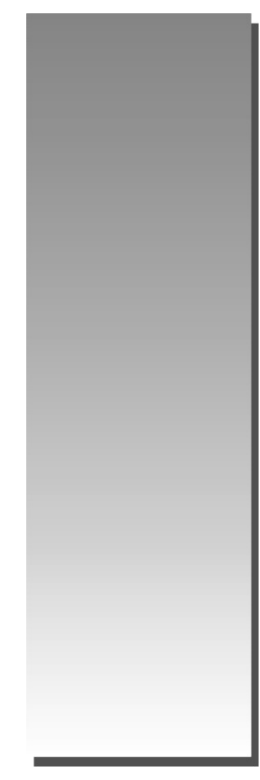

Mg. Enrique Huaco Pastor*

enrique_huaco@yahoo.com

\section{RESUMEN}

La Universidad Nacional Mayor de San Marcos, así como otras instituciones y empresas, una buena parte de ellas, en el Perú y en el extranjero, en el siglo XX asumieron una estructura organizativa formal inscrita en el modelo de organización burocrático que postuló Max Weber, y aún en la actualidad coexisten con otros modelos organizativos, técnicamente denominados modelos tradicionales. Los significativos cambios económicos, sociales, políticos y tecnológicos producidos en las últimas décadas en el mundo han generado que el modelo organizativo burocrático con sus mejores cualidades, haya sido superado técnicamente por otros modelos modernos flexibles y que pueden hacer frente con mayor eficiencia y eficacia a los nuevos desafíos de los entornos local, sectorial, nacional y global que se inserta en una dinámica discontinua, de incertidumbre y excesivamente competitiva.

Hoy, en un mundo globalizado, en la era del conocimiento, en el que la que a la racionalidad de la eficiencia y eficacia se suma la calidad, productividad y la competitividad juega un papel importante la estructura organizativa y hasta de supervivencia; es imprescindible vislumbrar que la gestión universitaria pública no esta ajena a dichos conceptos y desafios, motivo por el cual la Universidad Nacional Mayor de San Marcos durante esta última década pasada ha buscado encarar estos retos para mejorar su servicio Calidad y Gestión Académica-Administrativa; sin embargo, los sistemas de gestión administrativas, y el sistema de la planificación no han podido generar aún cambios significativos en la gestión.

La Universidad Nacional Mayor de San Marcos, para el año 2021, deberá asumir un entorno más dinámico, en la que las tendencias de las mutaciones en la ciencia, tecnología, innovación, así como los cambios políticos, económicos, sociales, ecológicos del Perú, al estar insertados con más fuerza en el entorno global, servirán de estímulo para generar en la universidad en forma intensiva, un rol de gran importancia y de trascendencia, como un centro académico y de investigación, capaces de producir conocimientos, ciencia y tecnologías con estándares competitivos internacionales sostenibles, en materias vinculadas a suministrar el soporte multidisciplinarios suficiente para el desarrollo económico, social, cultural y responsabilidad social en el ámbito nacional, con reconocimiento en el ámbito continental y global.

* Licenciado en Administración, Egresado de Maestría en administración, Docente Asociado de la Facultad de Ciencias Administrativas de la UNMSM. 


\section{INTRODUCCIÓN}

En estos últimos años, en el mundo y en el Perú, se han producido acelerados cambios en la ciencia y tecnología con impacto en el entorno económico, social, jurídico y político, situación problemática que demandan su reforma y modernización del aparato público y, en particular, de las universidades públicas que impulsen y se involucren en el desarrollo sostenible y armónico del Perú.

El proyecto de investigación plantea analizar el modelo de organización burocrático de la UNMSM, revelando las distorsiones y anomalías que provienen de sus efectos, identificando sus limitaciones y potencialidades con el objeto de que, mediante enfoques y criterios modernos de la tecnología organizativa, proponer nuevas estructuras organizativas para el periodo temporal de 2011 a 2021, que contribuyan a la reforma y modernización del modelo estructural organizativo que hagan viable una producción de servicios con estándares de productividad y calidad para los alumnos, usuarios y beneficiarios, promoviendo el desarrollo de la colectividad universitaria, así como coadyuve al desarrollo económico y social y al mejoramiento de la calidad de vida del país.

\section{MÉTODOS Y MUESTRA}

\section{MÉTODO}

La investigación será de tipo descriptivo - explicativo.

La metodología de investigación que se empleará permitirá lograr la demostración y contratación de las hipótesis correspondiendo a la metodología descriptiva - explicativa.

\section{MUESTRA}

La investigación se concentrará en el diseño y estructura organizativa formal de la universidad, expresada en los documentos normativos de gestión y de entrevistas mediante cuestionarios adecuadamente estructurados, así como efectuando un berchmarking con algunas universidades prestigiosas.

\section{HIPÓTESIS}

El modelo organizativo formal de la universidad, por su naturaleza burocrática, genera distorsiones originadas por sus características estructurales, conformando un modelo organizativo con estructuras formales ya superadas por otras formas de organización,que la prospectiva exige que para hacer frente a los desafios de la globalización, competividad e innovación, haciendo que surga la necesidad de proponer modelos de estructuras organizativas con estándares de productividad y competitividad satisfactorios, lo cual nos permite la interconexión institucional nacional y global, así como con la sociedad.

\section{MARCO LEGAL}

La Ley Marco de la Modernización del Estado, Ley 27658, que declara al Estado peruano en proceso de modernización en sus diferentes instancias, dependencias, entidades, organizaciones y procedimientos, con la finalidad de mejorar la gestión pública y construir un Estado democrático, descentralizado y al servicio del ciudadano.

LEY DE SIMPLIFICACIÓN ADMINISTRATIVA LEY 25035

Las funciones que desarrolla la administración pública están sujetas a los siguientes principios generales:

1. La presunción de veracidad, que rige en las relaciones de aquella con sus funcionarios y servidores y con el público, y que consiste en suponer que las personas dicen la verdad.

2. La eliminación de las exigencias y formalidades cuando los costos económicos que ellas impongan sobre la sociedad, excedan los beneficios que le reportan.

3. La desconcentración de los procesos decisorios a través de una clara distinción entre los niveles de dirección y los de ejecución.

4. La participación de los ciudadanos en el control de la prestación de los servicios por parte de la administración pública, y en la prestación misma de los servicios.

\section{DECRETO SUPREMO № 025-2010-PCM}

\section{POLÍTICAS NACIONALES EN MATERIA DE SIMPLIFICACIÓN ADMINISTRATIVA}

Entre las políticas nacionales, establece como principio a la gestión basada en procesos. En los procedimientos administrativos, y para la prestación de servicios en exclusividad, debe darse relevancia a la optimización de procesos superando las desventajas de los modelos de organización que puedan tener, basados en estructuras rígidas y jerárquicas, la operación por funciones o manuales bajo una óptica tradicional. 


\section{LEY DEL SILENCIO ADMINISTRATIVO}

\section{LEY № 29060 ARTÍ́CULO 1.- OBJETO DE LA LEY}

Los procedimientos de evaluación previa están sujetos a silencio positivo, cuando se trate de algunos de los siguientes supuestos:

a) Solicitudes cuya estimación habilite para el ejercicio de derechos preexistentes o para el desarrollo de actividades económicas que requieran autorización previa del Estado.

b) Recursos destinados a cuestionar la desestimación de una solicitud o actos administrativos anteriores.

\section{EXPOSICIÓN DE RESULTADOS}

Observaciones a la estructura organizativa de la UNMSM

La estructura organizativa de la UNMSM tiene la armazón, por sus características estructurales, de un modelo de corporación, debiendo ser abordado su estudio en sus tres dimensiones estratégicas: la corporativa, o sea, la institución como un todo, las unidades de servicios, o sea, de las distintas facultades y, finalmente, la estructura funcional, es decir, los instrumentos normativos de gestión representados por el estatuto, reglamento general, reglamento de organización y funciones (ROF) y el manual de organización y funciones (MOF),

La estructura organizativa de la UNMSM está diseñada con el criterio clásico de departamentalización funcional y divisional, incorporando un conjunto de normas que le otorgan un carácter impersonal, con lo cual se asume un modelo de organización con características burocráticas.

El modelo burocrático se expresa en una estructura medianamente compleja, que se muestra específicamente por el buen número de unidades organizativas u órganos y de cargos en la construcción de la organización.

Así también, el modelo se manifiesta por haber incorporado a la estructura organizativa una formalidad y estandarización relativa, por el estatuto, los reglamentos, manuales y directivas instituidas que condicionan a un comportamiento de sus integrantes a la norma y/o regla, limitando la amplitud de discrecionalidad, así como el contraer al servidor a las tareas que corresponde al cargo y/o puesto en el que ha sido nombrado, y a que el servidor no acceda y se resista a ser reubicado en otro cargo.
La estructura organizativa establece el elemento de la unidad de mando, con una cadena de mando y de jerarquía suficientemente instituida, reforzada por la centralización de la toma de decisiones fundamentales en el vértice de la estructura organizativa, lo que provoca que la conclusión de muchos de los actos administrativos deban resolverse con la toma de decisiones en los niveles de dirección y/o de jefatura.

El modelo organizativo piramidal de la universidad normativamente establece autonomía de gestión para las distintas facultades, no obstante la desconcentración administrativa asumida por la UNMSM no implica una integral descentralización $\mathrm{y}$, al estar inserta en el modelo organizativo burocrática, impone una centralización en los aspectos relevantes de la gestión a los niveles centrales o sea, correspondiente a la alta dirección y dirección conformadas en el ámbito operativo de gestión por el rectorado y vicerrectorados, direcciones generales; así como por los órganos de asesoría y de apoyo del nivel central.

Estas anomalías que estamos exponiendo afectan a la gestión al extender, solo espacial y funcionalmente, la estructura organizativa, lo que conlleva a tener procedimientos extensos $\mathrm{y}$ engorrosos, que tienen que transitar por las distintas unidades operativas comprendidas en los distintos niveles organizativos y a través de las distintas jerarquías o cadenas de mando en el interior organizativo de las facultades. Así mismo en la parte central de la universidad, el órgano del rectorado presenta un tramo de control atípico, que representa el atributo administrativo de realizar la planificación, la transmisión de disposiciones y/u órdenes, así como de evaluación que corresponden a los órganos de jefatura para los órganos con relación de dependencia por el excesivo número de órganos de consultaría, apoyo, postgrado asesorías, y de las facultades que mantienen una relación de dependencia directa con el rectorado, órganos dependientes que exceden al número de treinta.

Los órganos de los vicerrectorados académicos y de investigación, también exhiben tramos de control amplios, al mostrar orgánicamente una relación de dependencia con siete y seis oficinas respectivamente.

Los órganos del rectorado y vicerrectorados orgánicamente deberían estar concentrados en liderar las funciones que corresponden a la esencia 
de la universidad, o sea, las funciones académicas, de investigación, de proyección y de extensión social; no obstante tienen adscritos otros órganos administrativos que sustraen formalmente su atención y prelación de las funciones sustantivas académicas, de investigación y proyección social.

La Oficina General de Planificación, que es el órgano de asesoría, también se muestra como atípico. Entre otras funciones le corresponden las propuestas de diseño de la organización formal y de racionalización administrativa. Según el cuadro de asignación de personal (CAP) está operando con veintiséis cargos; en ese número, presentan tres asesores, un técnico administrativo, un especialista y veintiún jefes, de los cuales veinte no tienen cargos y personal que tengan relación de dependencia con esas jefaturas.

La autoridad formal de los órganos de dirección, línea, asesoría y apoyo a la UNMSM, no está claramente delimitada, igualmente en la delegación, presentando en ambos casos una desproporción entre la delegación de funciones con la autoridad formal inserto en los órganos.

La Oficina General de Asesoría Jurídica tiene trece profesionales, de los cuales siete tienen el cargo de jefes, un asesor y la diferencia son asistentes o especialistas. En la cobertura de los asuntos jurídicos cubren los aspectos laborales, civiles, administrativos, de transparencia y penales. Observamos que esta última jefatura tiene dos profesionales a tiempo completo, careciendo la estructura organizativa el cargo de un procurador.

Los órganos de apoyo presentan una estructura excesivamente arborizada, comprenden 19 oficinas de distinta naturaleza funcional, entre ellos, la clínica universitaria y la unidad de fincas.

Los órganos de línea están principalmente representados por las diferentes facultades, además la escuela de post grado, Instituto Porras Barrenechea, seminario de Historia Rural Andina, Museo de la Historia Natural, Jardines de Botánica y Jardín Ecológico, mostrando una ancha extensión horizontal estructural, haciendo inviable un tramo de control que sea estructuralmente eficaz y eficiente.

\section{Apreciación genérica a los Instrumentos Nor- mativos de Gestión de la Universidad Nacional Mayor de San Marcos}

La Facultad de Ciencias Contables forma parte de la UNMSM, que se inscribe en la Ley N. 23733 de Universidades en el Perú. En su artículo primero señala que "están integradas por profesores, estudiantes y graduados. Se dedican al estudio, la investigación, la educación y la difusión del saber y la cultura, y a su extensión y proyección sociales. Tienen autonomía académica, económica normativa y administrativa, dentro de la ley."

Igualmente en el artículo segundo señala que "Son fines de las universidades:

a) Conservar, acrecentar y transmitir la cultura universal con sentido crítico y creativo, afirmando preferentemente los valores nacionales.

b) Realizar investigación en las humanidades, las ciencias y las tecnologías y fomentar la creación intelectual y artística.

c) Formar humanistas, científicos y profesionales de alta calidad académica, de acuerdo con las necesidades del país, desarrollar en sus miembros los valores éticos y cívicos, las actitudes de responsabilidad y solidaridad nacional y el conocimiento de la realidad nacional, así como la necesidad de integración nacional, latinoamericana y universal.

d) Extender su acción y sus servicios a la comunidad, y promover su desarrollo integral, y

e) Cumplir las demás atribuciones que les señalen la Constitución, la ley y su estatuto.

La ley también indica que las universidades se rigen en su actividad por los siguientes principios:

a) La búsqueda de la verdad, la afirmación de los valores y el servicio a la comunidad.

b) El pluralismo y la libertad de pensamiento, de crítica de los valores y el servicio a la comunidad.

c) El pluralismo y la libertad de pensamiento, de crítica, de expresión y de cátedra con lealtad a los principios constitucionales y a los fines de la correspondiente universidad,

d) El rechazo de toda forma de violencia, intolerancia, discriminación y dependencia,

La organización de la Universidad Nacional Mayor de San Marcos está regulada por Ley Universitaria N.. 23733, el Estatuto, el Reglamento General y el Reglamento de Organización y Funciones y las facultades del Manual de Organización y Funciones, (MOF), Cuadro para la Asignación de Personal (CAP) y Texto Único de Procedimientos Administrativos (TUPA). 
Estos instrumentos de gestión normalizan y consolidan una estructura organizativa superada por otros modelos organizacionales, como la conformada por procesos, o sea, de una organización por procesos.

\section{CONCLUSIONES}

1. Estructura organizativa que muestra la organización formal de la UNMSM presenta serias anomalías de naturaleza estructural.

2. Estructura organizativa formal esta desfasada en el cumplimiento de las exigencias técnicas administrativas y jurídicas que demandan el entorno, y al Estado, al no estar comprendidas en la modernidad de la gestión pública.

3. El modelo organizativo que ha asumido la UNMSM, técnicamente puede responder a épocas pasadas, hoy en día el diseño de las organizaciones se orienta a lograr la estructura organizativa que tenga la racionalidad de insertar en ella las siguientes cualidades: eficiencia, eficacia, productividad, calidad, competitividad e innovación.

4. La estructura organizativa formal tiene un empleo marginal de las tecnologías de la información, comunicaciones, telecomunicaciones e informática, debiendo dar prioridad en los campos académicos, investigación y proyección social.

5. La normativa sobre organización expresada en los instrumentos normativos de gestión, es frondosa, repetitiva, generadora de duplicidades e indefiniciones, requiriendo su actualización y modernización.

6. La universidad orienta gran parte de su organización hacia el establecimiento de funciones o tareas que no apoyan la consecución de sus fines esenciales. La función de gobierno se encuentra recargada con funciones y responsabilidades administrativas operativas que deberían ser delegadas.

7. La asignación de funciones y creación de unidades orgánicas no obedece a criterios técnicos. En muchos casos las funciones administrativas se orientan a potenciar actividades rutinarias y que no generan valor ni desarrollan procesos de modernización.

8. Las funciones o tareas de personal, logística y finanzas son más formales que impulsores de mejoramiento organizativo.
9. Las facultades y áreas operativas han desarrollado soportes administrativos paralelos a los mecanismos administrativos formales, en algunos casos presentan deficiencias administrativas.

\section{PROPUESTA DEL NUEVO DISEÑO DE ORGANIZACIÓN FORMAL DE LA UNMSM}

La Universidad Nacional Mayor de San Marcos, al haberse ubicado en la era del conocimiento, en los años próximos deberá iniciar el camino de la asunción de un rol estratégico, de diagnosticar y de contar con un conjunto de propuestas, orientando su transformación a una universidad comprometida con una formación académica de investigación de mayor calidad, conducente a lograr la acreditación universitaria, asumiendo como desafíos la transferencia e innovación tecnológica dentro de una perspectiva de creatividad de avanzada, en los cauces multidisciplinarios y transdisciplinaria, con la actualización de las mallas curriculares de las carreras profesionales actuales, así como dentro del contenido del análisis prospectivo de los requerimientos de diseño e implementación de nuevas carreras profesionales que exigen el desarrollo nacional, el entorno globalizado y la competitividad.

La nueva estructura organizativa deberá asumir un modelo organizativo horizontal, flexible, con orientación comprometida con los estamentos de la comunidad universitaria (alumnos, egresados, profesores, autoridades, trabajadores) así como con la sociedad y sus organizaciones públicas y privadas insertas, que lo especificamos en el párrafo próximo.

El compromiso de la UNMSM al año 2021, fundamentalmente, además de formar profesionales idóneos en sus competencias y competitivos en el escenario nacional y global, es proyectar los conocimientos y su puesta en valor, transfiriéndolos a la sociedad peruana para que la producción de bienes y servicios y su exportación tengan cada vez mayor valor agregado y que concurran a mejorar la calidad de vida de la población peruana, en la hora presente, además de impulsar y reforzar sus inherentes competencias de la universidad, será la de incorporar a la universidad de observatorios científicos y tecnológicos, centros de investigación desarrollo de plataformas tecnológicas de última generación, centros de producción, centros de asesoría y consultaría en aspectos 
multidisplinarios, incubadoras de empresas y de organizaciones, talleres de conocimientos, que impliquen un despegue significativos de las ventajas comparativas que nos ofrece la naturaleza, a las ventajas competitivas generadas por la ciencia, tecnología e innovación, que la universidad se inserte intensivamente en la economía global del conocimiento, que promueva y actué de soporte científico y tecnológico a la producción y productividad con tecnología avanzada, compatible con los estándares internacionales de los países del primer mundo

La UNMSM, en su nueva estructura organizativa, deberá articular e interrelacionarse en redes dinámicas con universidades de prestigio nacionales y extranjeras, así como las organizaciones públicas y privadas del Estado peruano.
El modelo organizativo se deberá involucrar en la necesidad de ampliar sustancialmente sus capacidades y competencias fundamentales, que representan su razón de ser: la académica, de investigaciones, proyección y extensión social, en la prestación de los servicios inherentes a una universidad en forma personalizada, a distancia y virtual (e-learning en sus modalidades sincrónica y asincrónica), con empleo intenso de las tecnologías de la información, comunicaciones, telecomunicaciones, telemática, así también con las tecnología de la gestión. Y por consiguiente acrecentar la capacidad de oferta de matrícula de estudiantes de pre y post grado, de servicios de proyección social, asesoría y consultoría a las organizaciones públicas y privadas de la sociedad peruana e internacional.

\section{PROPUESTA DE NUEVA ESTRUCTURA ORGANIZATIVA UNMSM}

\section{UNIVERSIDAD : SEDE CENTRAL}

Conducir la gobernabilidad de la UNMSM, dinamizando sus competencias fundamentales: en lo Académico, Investigación y Proyección y extensión Social

\begin{tabular}{|l|}
\hline 01 ÓRGANOS DE ALTA DIRECCIÓN \\
\hline 01.1 LA ASAMBLEA UNIVERSITARIA \\
\hline 01.2 EL CONSEJO UNIVERSITARIO \\
\hline 01.3 EL CONSEJO DE FACULTAD \\
\hline 01.4 RECTORADO Y ÓRGANOS DE APOYO DEPENDIENTES \\
\hline 01.4.1 Oficina de Secretaria Administrativa \\
\hline 01.4.2 Oficina de Coordinación Técnica \\
\hline 01.4.3 SECRETARÍA GENERAL \\
\hline 01.4.3.1 Oficina de Archivo Central \\
\hline 01.4.3.2 Oficina de Secretaria Administrativa \\
\hline 01.4.4 OFICINA GENERAL DE IMAGEN INSTITUCIONAL \\
\hline 01.4.4.1 Oficina de Comunicaciones \\
\hline 01.4.4.2 Oficina de Actividades Institucionales \\
\hline 01.4.5 OFICINA GENERAL DE COOPERACIÓN Y RELACIONES INTERINSTITUCIONALES \\
\hline 01.4.5.1. Oficina de Convenios y Relaciones Interinstitucionales \\
\hline 01.4.5.2 Oficina de Cooperación Técnica Internacional \\
\hline 01.5 VICERRECTORADO ACADÉMICO Y ÓRGANOS DEPENDIENTES \\
\hline 01.5.1 Oficina Administrativa \\
\hline 01.5.2 CENTRO PREUNIVERSITARIO \\
\hline 01.5.2.1 Consejo Ejecutivo \\
\hline 01.5.2.2 Dirección Ejecutiva \\
\hline 01.5.2.3 Dirección Académica \\
\hline
\end{tabular}




\begin{tabular}{|c|}
\hline 01.5.2.4 Dirección Administrativa-Financiera \\
\hline 01.5.3 OFICINA GENERAL DEL SISTEMA DE BIBLIOTECAS Y BIBLIOTECA CENTRAL \\
\hline 01.5.3.1 Oficina de Automatización \\
\hline 01.5.4 SISTEMA ÚNICO DE MATRÍCULA (SUM) \\
\hline 01.5.5 CENTRO DE PRODUCCIÓN EDITORIAL E IMPRENTA (CEPREDIM ) \\
\hline 01.5.6 CENTRO DE PRODUCCIÓN LIBRERÍA Y DISTRIBUCIÓN (CENPROLID) \\
\hline 01.5.7 CENTRO DE PRODUCCIÓN FONDO EDITORIAL \\
\hline 01.6 VICERRECTORADO DE INVESTIGACIÓN Y ÓRGANOS DEPENDIENTES \\
\hline 01.6.1 Oficina Administrativa \\
\hline 01.6.2 Oficina de Registro de Actividades de Investigación ( RAIS ) \\
\hline 01.6.4. CONSEJO SUPERIOR DE INVESTIGACIÓN ( CSI ) \\
\hline 01.6.4.1 Oficina de Fomento a la Investigación \\
\hline 01.6.4.2 Oficina de Fomento a la Formación Científica \\
\hline 01.6.4.3. Oficina de Información Científica y Tecnológica \\
\hline 01.6.5 CONSEJO DE TRANSFERENCIA E INNOVACIÓN ( CTI ) \\
\hline 01.6.5.1 Oficina de Oferta Tecnológica \\
\hline 01.6.5.2 Oficina de Patentes y Derechos de Propiedad Intelectual \\
\hline 01.6.6 CONSEJO DE GESTIÓN DE INVESTIGACIÓN ( CGI ) \\
\hline 01.6.6.1 Oficina de Proyectos, Gestión y Promoción \\
\hline 01.6.6.2 Oficina de Planeamiento y Desarrollo \\
\hline 02 ÓRGANOS CONSULTIVOS \\
\hline 02.1 COMISIONES DE LA ASAMBLEA UNIVERSITARIA \\
\hline 02.2 COMISIONES DEL CONSEJO UNIVERSITARIO \\
\hline 04.3 OFICINA CENTRAL DE CALIDAD ACADÉMICA Y ACREDITACIÓN \\
\hline 04.3.1 Oficina de Promoción e Investigación de la Calidad \\
\hline 04.3.2 Oficina de Evaluación de la Calidad Universitaria \\
\hline 04.3.3 Oficina de Gestión de la Calidad \\
\hline 04.3.4 Oficina de Capacitación \\
\hline 03 ÓRGANOS DE CONTROL \\
\hline 03.1 ÓRGANO DE CONTROL INSTITUCIONAL \\
\hline 03.1.1 Oficina de Planeamiento y Asesoría Técnica \\
\hline 03.1.2. Oficina de Auditoría Financiera \\
\hline 03.1.2 Oficina de Auditoría de Gestión \\
\hline
\end{tabular}

\section{UNIVERSIDAD : ÓRGANOS DE ASESORÍA}

Se propone que los órganos de asesoría sean descentralizados administrativamente, dotándoles de capacidades de autonomía administrativas, adscrita a los órganos de apoyo y de dirección de la UNMSM, con atribuciones para captar el capital humano de las distintas facultades de la universidad, y ofertar servicios de asesoría y consultaría en diferentes materias empresariales, sociales, jurídicas y políticas a la sociedad. 


\begin{tabular}{|l|}
\hline 04 ÓRGANOS DE ASESORAMIENTO \\
\hline 04.1 OFICINA GENERAL DE PLANIFICACIÓN \\
\hline 04.1.1 Oficina de Planes y Programas \\
\hline 04.1.2 Oficina de Presupuesto \\
\hline 04.1.3 Oficina de Racionalización \\
\hline 04.1.4 Oficina de Estadística e Informática \\
\hline 04.1.5 Oficina de Coordinación de Centros de Producción \\
\hline 04.2 OFICINA GENERAL DE ASESORÍA LEGAL \\
\hline 04.2.1 Oficina de Asuntos Administrativos \\
\hline 04.2 .2 Oficina de Asuntos Civiles \\
\hline 04.2 .3 Oficina de Asuntos Penales \\
\hline 04.2 .4 Oficina de Asuntos Laborales y Seguridad Social \\
\hline 04.2 .5 Oficina de Transparencia y Acceso a la Información. \\
\hline
\end{tabular}

\section{UNIVERSIDAD : ÓRGANOS DE APOYO}

Se propone que el órganos de apoyo se desconcentre administrativamente, debiendo adoptar una estructuración por procesos, liderada por la Dirección General de Administración (DGA)

\begin{tabular}{|l|}
\hline 05 ÓRGANOS DE APOYO \\
\hline 05.1 DIRECCIÓN GENERAL DE ADMINISTRACIÓN ( DGA ) Y ÓRGANOS DEPENDIENTES \\
\hline 05.1.1. Oficina Administrativa \\
\hline 05.1.2. Oficina de Control Previo y Fiscalización \\
\hline 05.1.3 OFICINA GENERAL DE BIENESTAR UNIVERSITARIO ( OGBU ) \\
\hline 05.1.3.1 Oficina de Alimentación \\
\hline 05.1.3.2 Oficina de Servicio Social \\
\hline 05.1.3.3 Oficina de Deportes y Recreación \\
\hline 05.1.3.4 Oficina de Coordinación Administrativa \\
\hline 05.1.4 OFICINA GENERAL DE ECONOMÍA \\
\hline 05.1.4.1 Oficina de Contabilidad \\
\hline 05.1.4.2 Oficina de Tesorería \\
\hline 05.1.4.3 Oficina de Abastecimiento \\
\hline 05.1.4.4 Oficina de Auto seguro de Accidentes \\
\hline 05.1.5 OFICINA GENERAL DE RECURSOS HUMANOS \\
\hline 05.1.5.1 Oficina de Gestión y Escalafón \\
\hline 05.1.5.2 Oficina de Remuneraciones y Obligaciones Sociales \\
\hline 05.1.5.3 Oficina de Pensiones y Beneficios Sociales \\
\hline 05.1.5.4 Oficina de Desarrollo y Bienestar Social \\
\hline 05.1.5.5 Oficina de Modernización e Informatización \\
\hline 05.1.6 OFICINA GENERAL DE INFRAESTRUCTURA UNIVERSITARIA \\
\hline 05.1.6.1 Oficina de Estudios y Proyectos \\
\hline 05.1.6.2 Oficina de Obras e Ingeniería \\
\hline 05.1.6.3 Oficina de Proyectos de Inversión \\
\hline
\end{tabular}


05.1.7.1 Oficina de Administración y Mantenimiento

05.1.7.2 Oficina de Transporte y Maestranza

05.1.8 UNIDAD DE FINCAS

05.1.9 CLÍNICA UNIVERSITARIA

\section{UNIVERSIDAD : ÓRGANOS DE LÍNEA}

Se propone la conformación de cinco Macro Decanatos:

- Ciencias Empresariales

- Ciencias Sociales

- Humanidades

- Ciencias de la Salud

- Ingenierías

Los mismos que agruparan las facultades relacionadas con su materia.

\begin{tabular}{|l|}
\hline 06 ÓRGANOS DE LÍNEA \\
\hline 06.1 FACULTADES \\
\hline 06.2 ESCUELA DE POST GRADO \\
\hline 07 OTROS ÓRGANOS ACADÉMICOS \\
\hline 07.1 OFICINA TÉCNICA DEL ESTUDIANTE (OTE ) \\
\hline 07.2 CENTRO CULTURAL \\
\hline 07.3 INSTITUTO RAÚL PORRAS BARRENECHEA \\
\hline 07.4 SEMINARIO DE HISTORIA RURAL ANDINA \\
\hline 07.5 MUSEO DE HISTORIA NATURAL \\
\hline 07.6 JARDINES BOTÁNICOS Y JARDÍN ECOLÓGICO \\
\hline
\end{tabular}

\section{DIRECTIVAS ESTRATÉGICAS}

La situación de globalización y la inserción de nuestro país implican un sustantivo cambio en las organizaciones del sector privado y público. En tal sentido, las propuestas establecidas en este estudio tienen como fundamentos las siguientes directivas estratégicas:

1. Adecuarse a un proceso de actualización y modernización de la UNMSM, que involucre la nueva visión del ámbito de la universidad peruana, así como el de adecuarse a la Ley de Modernización del Estado, que declara al Estado peruano en proceso de modernización en sus diferentes instancias, dependencias, entidades, organizaciones y procedimientos, con la finalidad de mejorar la gestión pública y construir un Estado democrático, descentralizado y al servicio del ciudadano.
2. Adecuarse a los cambios producidos en los nuevos modelos organizativas, con la horizontalizacion, la estructura por procesos y concentrado en el corazón de competencias funcionales de la UNMSM, representado por la misión académica, la misión de investigación y la misión de la proyección y extensión social.

3. Dicha adecuación deberá estar orientada a la búsqueda de una universidad capaz de lidiar con una estructura más concentrada en sus competencias esenciales, pero más inductora y articuladora en el marco de la globalización y las interacciones de la economía el Perú y del mundo.

4. La necesidad de absorber los cambios y los retos que nos plantean, las ciencias, la tecnología y la innovación a nivel mundial, y la vinculación estrecha con las actividades productivas nacionales. 
5. El reforzamiento de la idea de eficiencia, eficacia, productividad y competitividad con el objetivo que se empleen apropiadamente sus siempre finitos recursos en las áreas que le son sustantivas.

6. La búsqueda de una infraestructura social, interna física y tecnológica, capaz de ser coherente a los estándares internacionales de manejo de establecimientos educativos superiores exitosos.

El diseño general que se muestra seguidamente brinda un análisis dinámico en el que se conjugan la organización y los procedimientos; y debe tener consecuencias en la dotación de recursos, a través de la supervisión y el control. La estructura organizativa deberá incluir una estructura desconcentrada y descentralizada con articulación de redes en la conformación interna de sus flujos de trabajo en lo que fuera pertinente en conectar a la universidad a la red macro de las universidades nacionales e internacionales; así también con organizaciones privadas y públicas nacionales e internacionales.

\section{DISEÑO GENERAL}

El modelo general se basa en el otorgamiento de un mejor estatus a la función de gobierno del rectorado, encargado de las funciones políticas y de orientación general y a las responsabilidades de los vicerrectores, encargados de gerenciar los campos funcionales asignados. El término "gerenciar" lo debemos entender como conseguir resultados apropiados en función de los fines y prioridades asignadas.

La asignación de un nuevo rol a la función de planificación. Antes del inicio de cada año, la universidad debería formular un planeamiento estratégico de sus actividades. En este documento, se debería conjugar la técnica de planeamiento con los lineamientos políticos del consejo universitario y rectorado, analizando los posibles escenarios políticos, económicos y sociales.

La desconcentración y reforzamiento de los aspectos académicos y operativos en las facultades y centros productivos, los cuales intervendrían fundamentalmente en los planes de acción, que contiene el planeamiento operativo técnico, mediante el cual cada facultad y centro operativo define.
La Oficina General de Planificación y la de Asesoría Jurídica, como órganos de asesoría asumirían un rol dinámico y con atribuciones de captar el capital humano de la UNMSM, para ampliar y extender la producción de servicios al interior de la Universidad y hacia las organizaciones públicas y privadas de la sociedad, estableciendo redes con universidades nacionales y extranjeras y con otras organizaciones afines y de soporte.

Consideramos que las propuestas que a continuación formulamos deben regirse por los criterios siguientes:

Coherencia con los fines de la Universidad. Es necesario que la estructura organizativa, las funciones o procesos y los recursos asignados a las diferentes áreas de la organización, reflejen las prioridades por atender los fines establecidos por la constitución y la ley a la universidad, tales como, la formación de pre y postgrado, la investigación científica y tecnológica y la extensión y proyección social. En este sentido, también deberá revisarse que funciones y tareas actuales consumen la mayor cantidad de esfuerzo y recursos y evaluar si estas están realmente generan valor agregado y están vinculadas a las competencias esenciales de la Universidad.

Reforzamiento de la función de gobierno. Es necesario que los órganos de gobierno de la universidad, efectivamente realicen funciones de "gobierno". Entendidas como las de establecer regulaciones y prioridades en función de una visión de mediano y largo plazo, dirigir la entidad, supervisar sus actividades, reglamentar y aprobar políticas universitarias, en general, ejercer las funciones de dirección política y administrativa. Esto implicaría por contraste que las tareas que no corresponden a la función de gobierno y se refieran a la aplicación de las políticas, prioridades y regulaciones sean ejercidas por los otros niveles de la universidad.

La estructura organizativa deberá otorgar a las unidades de gobierno, del rectorado y vice rectorados, con especial énfasis en una mayor amplitud en la toma de las decisiones fundamentales, debiendo descongestionarse de competencias de gestión operativa y concentrarse en aquellas de gestión estratégica.

Reforzar la función de planificación universitaria. Es necesario que la estructura orgánica 
refleje la necesidad de establecer estrategias de adaptación permanente al entorno. En un mundo como el actual, en el cual el cambio es la constante, se hace necesario establecer funciones vinculadas al análisis prospectivo, que nos permita establecer los escenarios posibles en los campos de la ciencia, la economía y la realidad nacional que deben tenerse en cuenta en el establecimiento de planes, proyectos y programas de acción para las áreas académicas y administrativas. El no tener establecida apropiadamente la función de planificación, priva a las autoridades de gobierno y a los ejecutores de herramientas de la mejora de la calidad de decisiones, así como también del presupuesto y el gasto en general. En lo organizativo, un proceso de adaptación permanente al cambio requiere que la estructura organizativa garantice flexibilidad y adaptabilidad al entorno, asumiendo competencias que demanda el desarrollo nacional, con eficiencia, eficacia, calidad y competitividad, articuladas a los sistemas universitarios nacionales y extranjeros mediante el establecimiento de redes

Reforzar los órganos de ejecución. La estructura de organización debe establecer una desconcentración y descentralización alta en las tareas de ejecución debiendo la Universidad asumir la conformación y la naturaleza organizativa tipo holding o conglomerado, conformada con unidades estratégicas de formación académica y Profesional, lo que implicara desconcentrar las atribuciones de decisiones de los órganos de gobierno para que su ejecución se efectué a través de las facultades que son las unidades organizativas de prestación de los servicios. Esta desconcentración se debe expresar no solo en lo académico, sino principalmente en lo administrativo como soporte de lo académico. Esta desconcentración no significa fragmentar la universidad, sino integrarla a través de la centralización de los sistemas de información que refuercen la función sustantiva de gobierno, a través de la supervisión y el control. La estructura organizativa deberá incluir una estructura de integración de redes en la conformación interna de sus flujos de trabajo en lo que fuera pertinente con los centros de producción, así como por el lado del entorno conectar a la Universidad a la red macro de las universidades nacionales.

\section{CARACTERÍSTICAS PRINCIPALES DEL NUEVO MODELO ORGANIZATIVO}

1. Organización desburocratiza, horizontal, flexible, adecuada a las exigencias derivadas de la modernización de las instituciones del Estado, con mejores capacidades formales para enfrentar el entorno de la nueva economía;

2. Incremento sustancial en la oferta académica de la enseñanza -aprendizaje, en las modalidades: presencial, a distancia y e-learning, así como constituir a la universidad como una organización inteligentes acreditada por estándares internacionales en sus competencias académicas y de investigación;

3. Aumento sustantivo en sus capacidades de la investigación, dado que la estructura organizativa captará y dinamizará al capital humano de la universidad, representado por los catedráticos de las distintas facultades;

4. Establecimiento de redes con universidades nacionales y extranjeras de prestigio, ampliando la cobertura de las competencias inherentes a la universidad;

5. Establecimiento de convenios con los gobiernos nacionales, regionales y locales, así como con las organizaciones del sector público y privado, constituyéndose la universidad en un soporte para el desarrollo nacional;

6. Incremento sustancial en el desarrollo de las capacidades incorporadas mediante plataformas tecnológicas de última generación, así como del desarrollo de infraestructura moderna;

7. Aumento sustantivo de los servicios de proyección y extensión social, desarrollando una mayor cobertura en los espacios geográficos del Perú;

8. Acrecentamiento en sus capacidades económicas financieras, que conllevará a que los integrantes de la comunidad universitaria mejoren sus remuneraciones. 


\section{BIBLIOGRAFÍA}

B. J. HOGDE, WP. Anthony, L.M. GALES. Teoría de la Organización. Un Enfoque Estratégico. 5. Edición Prentice Hall, México, 1999.

KAST E., Fremont y ROSENZWEING, James E. Administración en las Organizaciones, Enfoque de Sistemas y Contingencias. 4. Edición Mc Graw-Hill. 1999.

KOONTZ, Harold y WIHRICH, Heinz. Administración. Una perspectiva global. Mc Graw Hill, 11.e edición, 1998.

MINTZTZBERG HENRY. Estructura Organizacional. Editorial Ariel. Barcelona - 2001.
MINTZHERG, HENRY. El Proceso Estratégico. Editorial Prentice Hall. Barcelona 2001.

PEPPARD y ROWLAND.- La Esencia de la Reingeniería en los Procesos de Negocios. Prentice Hall Hispanoamericana, México, 1997.

STONER, James y GILBERT, Freeman. Administración: Mc Graw Hill, 7.a edición, 1998.

Richar L. Daft. Teoría y Diseño Organizacional. 8. Edición Thomson, México, 2005.

HUACO PASTOR, Enrique. Guía Técnica para el Diseño Organizacional. Editorial San Marcos, Lima, 2005. 